\title{
PHOTOEMISSION TESTS of a Pb/Nb SUPERCONDUCTING PHOTOINJECTOR
}

\author{
J. Smedley, T. Rao BNL, Upton, USA \\ P. Kneisel, TJNAF, Newport News, USA \\ J. Sekutowicz, J. Iversen, D. Klinke, D. Kostin, W. Möller, A. Muhs, DESY, Hamburg, Germany \\ R. Lefferts, A. Lipski, SUNY, Stony Brook, USA
}

\begin{abstract}
We report recent progress in the development of a hybrid lead/niobium superconducting RF (SRF) photoinjector. The goal of this effort is to produce an injector with the SRF properties of a niobium cavity along with the superior quantum efficiency (QE) of a lead photocathode. Quantum efficiency measurements have been performed on an RF cavity with an electroplated lead plug as the photocathode. The effect of the laser on the RF performance of the cavity was characterized. Further measurements of the cavity RF performance are reported in these proceedings [6].
\end{abstract}

\section{INTRODUCTION}

An injector capable of delivering moderate average current $(\sim 1 \mathrm{~mA})$ along with significant peak current $(\sim 100$ A) is needed for a variety of applications, including nextgeneration energy recovery linac based free electron lasers $[1,2]$. Superconducting cavity technology is uniquely suited to this task, as superconducting cavities are capable of supporting high accelerating gradients to minimize space charge problems at high peak currents, while avoiding the cooling issues inherent in high average current normal conducting RF injectors. A superconducting photoinjector has its own challenges principally, how to introduce the photocathode into the superconducting cavity. An innovative solution to this has been developed by Forschungszentrum Rossendorf [3], utilizing an RF choke joint to allow a normal conducting cathode to be used in a superconducting cavity. We explore a second option - using the superconducting wall of the cavity as a photocathode. Previous work has demonstrated the feasibility of the concept by using the $\mathrm{Nb}$ back wall of the cavity itself as the photocathode. However, the low QE of $\mathrm{Nb}$ precludes this application [4]. For this reason, we concentrate on superconducting lead, which has been shown to provide roughly an order of magnitude higher QE compared to $\mathrm{Nb}$ [7] at room temperature with DC bias. The DC work investigated various methods of producing lead films on a niobium substrate, and concluded that electroplating and arc-deposition were attractive approaches for lead film based cathodes in a photoinjector.

This paper will describe measurements of the QE of a 3 $\mu \mathrm{m}$ lead film electroplated on a niobium plug. The $7 \mathrm{~mm}$ diameter plug matches a hole in the center of the backplate of a $1 / 2$ cell, $1.42 \mathrm{GHz}$ SRF cavity. In this arrangement, the photocathode can be arc deposited or electroplated (a technically difficult process in a normal $1 / 2$ cell) separately, and superconducting cavity and the photocathode can be cleaned independently, avoiding the exposure of the cathode to chemicals used for cleaning the SRF cavity. A second hybrid injector has also been tested recently [5]. This $1 / 2$ cell, $1.3 \mathrm{GHz}$ cavity had a $4 \mathrm{~mm}$ diameter lead spot plated on the center of the back wall via arc deposition. The details of the RF measurements on both of these cavities are reported elsewhere [6].

\section{LAYOUT}

A cryostat in the Vertical Test Area (VTA) at Jefferson Lab was used for these measurements. The cryostat is set into the floor of the VTA - the bottom of the cryostat is almost 3 meters below the floor. A retractable radiation shield covers the cryostat. The internal volume of the radiation shield above the cryostat top flange is $\sim 1 \mathrm{~m}^{3}$. This space was used to house the photocathode laser and the transport optics. The cryostat is capable of cycling from room temperature to $2 \mathrm{~K}$ to room temperature in 8 hrs. The cryostat enclosure has RF sources for 0.7 to 2 $\mathrm{GHz}$, as well as RF diagnostics.

\section{Laser and Optical Layout}

A KrF excimer laser (GAM EX5) was used to both clean the cathode and extract photoelectrons. This laser is capable of producing $\sim 6 \mathrm{~mJ}$ of $248 \mathrm{~nm}$ light per pulse, with a pulse duration of 5.3 ns FWHM and a variable pulse repetition frequency of $20-250 \mathrm{~Hz}$. The laser and the transport optics were mounted on a breadboard bolted to the cryostat top flange. The beam path on the breadboard was folded to allow 1:1 relay imaging of an iris onto the cathode using a $1.5 \mathrm{~m}$ lens. The optical path length was almost $6 \mathrm{~m}, 3 \mathrm{~m}$ prior to the lens, and $3 \mathrm{~m}$ from the lens to the cathode in the cryostat. The optical transport efficiency was $55 \%$ through three turning mirrors, the lens, and the input flange of the cryostat.

\section{Laser Cleaning}

Higher energy from the excimer laser was used to improve the QE of the cathode by driving off surface contaminants. Previous work on lead has established an optimum energy density of $\sim 0.2 \mathrm{~mJ} / \mathrm{mm}^{2}$ for cleaning lead to maximize the $\mathrm{QE}$ while avoiding damage to the coating [7]. Laser cleaning was performed with the full beam area, resulting in a cleaned area of $5 \times 4 \mathrm{~mm}^{2}$ on the cathode. The cathode was irradiated for 200 seconds $(30 \mathrm{k}$ shots at $150 \mathrm{~Hz}$ ). 


\section{Cavity Orientation}

For RF cavity measurements, the cavity was initially mounted with the sealed end of the cavity up, as shown in Figure 1. This orientation was used to prevent debris from falling on the cavity during testing.

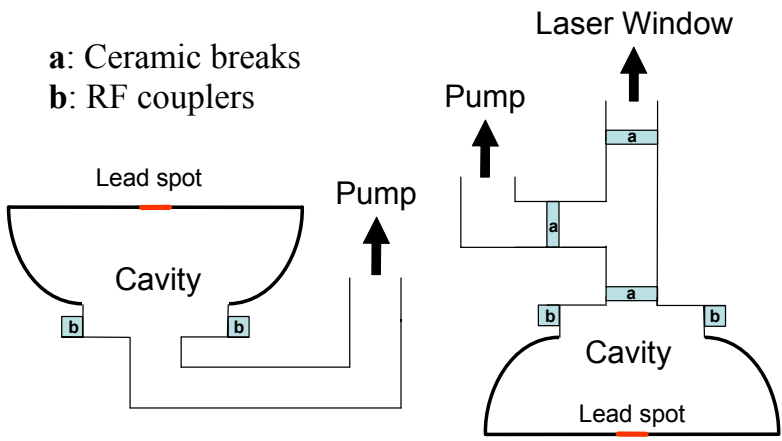

Figure 1: (left) Cavity orientation for RF measurements (right) Cavity orientation for QE measurements.

For QE measurements in the RF cavity, the cavity was mounted with the exit iris up to allow the laser to hit the cavity back wall. The cavity was electrically isolated from the laser tranport tube with a ceramic break. DC isolators were used on the RF input and pickup cables. The outer wall of the cavity was connected via an electrical feedthrough on the top flange of the cryostat to a picoammeter (Keithley 6485). The last $1.5 \mathrm{~m}$ of the laser transport tube was isolated from ground as well. This allowed a DC bias of $1 \mathrm{kV}$ to be used on the tube, preventing secondary electrons from the tube from reaching the cavity.

\section{Current Measurements}

The current leaving the RF cavity was measured on the picoammeter. A baseline was taken without the laser to establish the "dark" (field-emission) current. For the photocurrent, a correction factor is required to account for the fact that the laser pulse duration of $5.3 \mathrm{~ns}$ covers many RF cycles at $1.42 \mathrm{GHz}$. A simulation was performed using Astra to determine the fraction of the electrons that could escape the cavity as a function of the peak electric field. The results are shown in fig. 2.

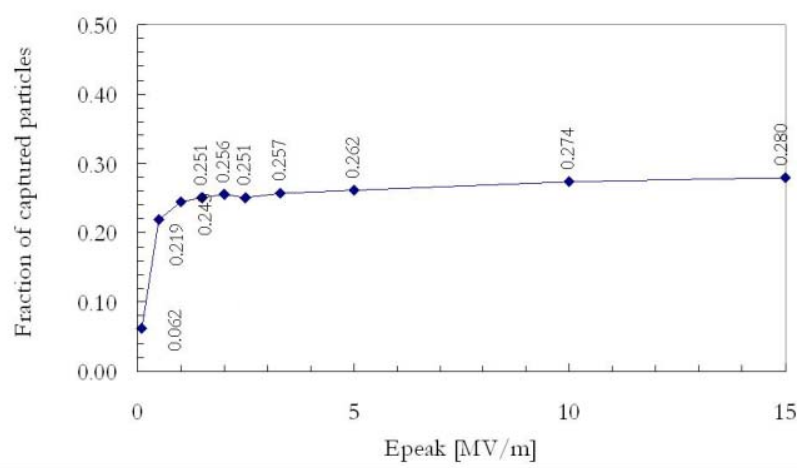

Figure 2: Fraction of photoelectrons which escape the cavity.

\section{RF CAVITY MEASUREMENTS}

The cleaning, preparation and RF performance of this cavity are the subject of a related paper [6]. In brief, the $\mathrm{Q}_{0}$ of the cavity with a solid niobium plug was $3 \times 10^{9}$ at 2 $\mathrm{K}$, and the peak field was over $40 \mathrm{MV} / \mathrm{m}$. With an electroplated lead plug, the low field $\mathrm{Q}_{0}$ was $6 \times 10^{9}$, and the peak field achieved was just under $40 \mathrm{MV} / \mathrm{m}$. The RF performance degraded significantly when the cathode was mounted right-side up for the QE measurements. In this configuration, significant conditioning was required to reach 4.5 MV/m, and the $\mathrm{Q}_{0}$ at low field was only $2.2 \times 10^{9}$. This degradation was likely caused by debris falling from the laser transport tube onto the cavity surface.

Figure 3 shows the QE of the electroplated lead plug before and after laser cleaning with energy densities of 0.01 and $0.15 \mathrm{~mJ} / \mathrm{mm}^{2}(0.2 \mathrm{~mJ} \& 3 \mathrm{~mJ}$, respectively). During all measurements, the laser was running with a repetition rate of $150 \mathrm{~Hz}$, with a pulse energy of between 1.5 and $5.8 \mu \mathrm{J}$ on the cathode. The QE did not depend on the laser pulse energy, indicating that space charge did not affect the results. The calculated QE includes the correction factor from fig. 2.

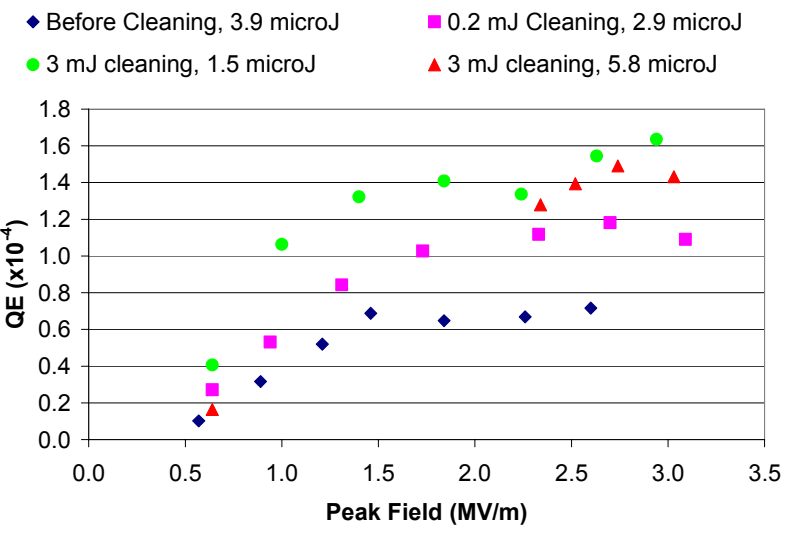

Figure 3: QE for electroplated lead plug. Laser pulse energy used for each measurement is listed in caption.

The pulse energies used for the QE measurements did not have an observable effect on the RF performance of the cavity. To observe a change in cavity $\mathrm{Q}$, the niobium wall of the cavity was irradiated with the maximum available laser pulse energy. The niobium wall was chosen to avoid damage to the lead coating. The magnetic field is somewhat higher at this location $(1-2 \mathrm{~cm}$ off of the cavity axis), thus any effect of localized breaking of Cooper pairs is expected to be more prevalent compared to on-axis irradiation. Figure 4 shows the effect of the full laser power $(2.9 \mathrm{~mJ})$ on the Q of the cavity (in a $5 \times 4 \mathrm{~mm}^{2}$ spot) as a function of the laser repetition rate. The $\mathrm{Q}_{0}$ was reduced by $20-30 \%$ compared to the "dark" value, but no evidence of a quench was observed. A pulse energy of $1 \mathrm{~mJ}$ was used on a smaller spot $(3 \mathrm{~mm}$ diameter), resulting in a $\sim 8 \%$ reduction in the $\mathrm{Q}$. In both cases, no clear dependence on the laser repetition rate is observed, indicating that the time scale for recovery of the broken Cooper pairs is much shorter than the time 
between pulses, as expected from theory [6]. As the laser energy density was comparable for both the $1 \mathrm{~mJ}$ and 2.9 $\mathrm{mJ}$ measurements, it is possible that the area of the wall with broken Cooper pairs is the dominant factor affecting the $\mathrm{Q}$.

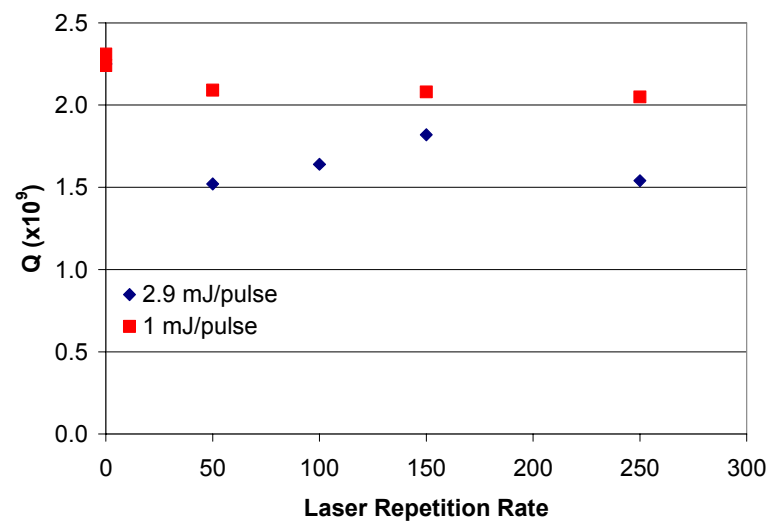

Figure 4: Laser-induced Q reduction.

\section{CONCLUSIONS}

A QE of $1.6 \times 10^{-4}$ at $248 \mathrm{~nm}$ has been measured for an electroplated lead photocathode in a niobium cavity. This $\mathrm{QE}$ is in good agreement with the $1.4 \times 10^{-4} \mathrm{QE}$ measured for electroplated lead at room temperature under $1 \mathrm{MV} / \mathrm{m}$ DC bias [7]. It is interesting to note that this QE requires very little laser cleaning to achieve, compared to DC measurements. It is possible that the RF processing required in the cavity reduces the level of surface contamination of the cathode, reducing the need for laser cleaning.

The lead coating does not significantly impact the RF performance of the cavity [6]. The laser striking the photocathode did not affect RF performance except at very high pulse energies. For pulse energies over $1 \mathrm{~mJ}$, a marginal reduction of the cavity Q was observed, but the cavity did not quench. The reduction of the $\mathrm{Q}$ depends on the laser pulse energy, but not on the repetition rate, and therefore not on the total power of the laser. It is expected that the time for Cooper pair recovery is less than $1 \mu \mathrm{s}$ [6]. In this case, for laser repetition rates up to $1 \mathrm{MHz}$, the laser should have little impact on the RF performance as long as the pulse energy is less than $1 \mathrm{~mJ}$ (corresponding to a bunch charge of $>25 \mathrm{nC}$ at the observed QE).

\section{ACKNOWLEDGMENT}

We would like to express our gratitude to colleagues at BNL, DESY and TJNAF from whom we received technical support.

This manuscript has been authored by Brookhaven Science Associates, LLC under Contract No. DE-AC0298CH10886 with the U.S. Department of Energy. The United States Government retains, and the publisher, by accepting the article for publication, acknowledges, a world-wide license to publish or reproduce the published form of this manuscript, or allow others to do so, for the United States Government purposes.

\section{REFERENCES}

[1] J. Sekutowicz et al., Phys. Rev. ST Accel. Beams 8, 010701 (2005)

[2] F. Gabriel et al., Nucl. Instr. and Meth. in Phys. Res. B 161-163, 1143 (2000)

[3] D. Janssen et al., Nucl. Instr. and Meth. in Phys. Res. A 507, 314 (2003)

[4] T. Rao et al, Nucl. Instr. and Meth. in Phys. Res. A562 22 (2006)

[5] J. Smedley et al., "QE Measurements of a Nb-Pb SRF Photoinjector", Proceedings of the 41st Advanced ICFA Beam Dynamics Workshop on Energy Recovery Linacs, Daresbury, UK, May 21-25 2007

[6] J. Sekutowicz et al., "Status of $\mathrm{Nb}-\mathrm{Pb}$ Superconducting RF-Gun Cavities", these proceedings

[7] J. Smedley et al., "Progress on Lead Photocathodes for Superconducting Injectors", Proceedings of 2005 Particle Accelerator Conference, Knoxville, Tennessee, 2598 (2005). 